\title{
Meta
}

Journal des traducteurs

Translators' Journal

\section{Otman, Gabriel (1996) : Les représentations sémantiques en terminologie, coll. "sciences cognitives", Paris, Masson, 216 p.}

\section{François Gaudin}

Volume 42, numéro 4, décembre 1997

URI : https://id.erudit.org/iderudit/002814ar

DOI : https://doi.org/10.7202/002814ar

Aller au sommaire du numéro

Éditeur(s)

Les Presses de l'Université de Montréal

ISSN

0026-0452 (imprimé)

1492-1421 (numérique)

Découvrir la revue

Citer ce compte rendu

Gaudin, F. (1997). Compte rendu de [Otman, Gabriel (1996) : Les représentations

sémantiques en terminologie, coll. "sciences cognitives", Paris, Masson, 216 p.]

Meta, 42(4), 729-732. https://doi.org/10.7202/002814ar

Ce document est protégé par la loi sur le droit d'auteur. L'utilisation des services d’Érudit (y compris la reproduction) est assujettie à sa politique d'utilisation que vous pouvez consulter en ligne.

https://apropos.erudit.org/fr/usagers/politique-dutilisation/
Cet article est diffusé et préservé par Érudit.

Érudit est un consortium interuniversitaire sans but lucratif composé de l’Université de Montréal, l’Université Laval et l’Université du Québec à Montréal. Il a pour mission la promotion et la valorisation de la recherche. https://www.erudit.org/fr/ 
- OTMAN, Gabriel (1996) : Les représentations sémantiques en terminologie, coll. «sciences cognitives», Paris, Masson, 216 p.

Comme son titre l'indique, l'ouvrage de Gabriel Otman, issu de sa brillante thèse de doctorat, ne peut être qu'un ouvrage utile. La sémantique reste le point faible de la terminologie. Sa publication permet donc aux étudiants de sciences du langage de disposer d'un ouvrage de référence, synthétique, clairement structuré et dont les thèses sont illustrées de très nombreux exemples. Ajoutons que la lecture en est agréable, grâce à une écriture ferme, qui évite tout pédantisme en n'oubliant pas d'être précise. Les annexes, le glossaire détaillé (pp. 185-202), une bibliographie fournie et l'indispensable index complètent cet ouvrage de réflexion et permettent d'en faire un manuel indispensable pour les étudiants intéressés à la terminologie, à la modélisation des connaissances et, plus largement, aux approches contemporaines de la sémantique.

La sémantique constitue la préoccupation principale de l'auteur. Or, la difficulté de la terminologie tient en ce qu'elle se penche sur des vocabulaires qui résistent, spécialisés, professionnels, énoncés dans des situations où l'implicite règne, ils émanent souvent de communautés linguistiques réduites et transnationales. Gabriel Otman s'est confronté à cette difficulté en décrivant le vocabulaire de l'intelligence artificielle (IA), qu'il utilise ici comme corpus.

L'objectif de l'auteur est de présenter «un modèle théorique de représentation formelle des unités terminologiques d'un domaine» (p. 1). Ce modèle, appelé «réseau sémantico-terminologique» (RST), doit être cohérent, représentatif des unités de discours et fonctionnel. Comme il convient, le premier chapitre permet à l'auteur d'installer le décor théorique dans lequél son argumentation va prendre place. N'hésitons pas à le ranger dans les auteurs post-classiques: il se pose lui-même en wüstérien éclairé et reprend à son compte l'équation concept $=$ terme (p. 14). Conscient des limites qu'impose un tel cadre, l'auteur les justifie par sa "problématique de modélisation à outrance» (p. 16). C'est un modeste. Certes, son approche ne se soucie pas de 
sociolinguistique, mais cette limitation, acceptée en connaissance de cause, s'avérera fructueuse par la suite.

La confrontation de la terminologie au domaine de l'IA conduit l'auteur à s'intéresser à la notion de «connaissances» (pp. 20-27) qu'il envisage dans une perspective évacuant consciemment l'histoire et l'évolution des savoirs. La connaissance considérée est essentiellement experte, sa répartition sociale n'est guère prise en compte. Le corpus linguistique sera, comme c'est la règle bien souvent, constitué de noms monosémiques. Le principe de monosémie, qu'il voit «constamment battu en brèche», est défendu par le recours aux domaines et, au sein d'un domaine, par l'analyse componentielle.

Modéliser les connaissances suppose préalablement de parvenir à les classer; le second chapitre est donc consacré aux systèmes de classification. En terminologie wüstérienne, la classification relève d'une logique documentaire fondée sur des domaines et des sous-domaines. La spécialisation conduit même à parler de «domaines d'application», voire de «micro-domaines» (p. 30). Il éclaire cette première approche par l'examen des relations prises en compte dans les thésaurus. L'auteur montre leur insuffisance pour une description sémantique. En revanche, dans les fortes pages qu'il consacre à la taxinomie (pp. 34-43), il la présente comme un exemple de construction langagière efficace organisant de très vastes secteurs du monde. Les théories des «taxilogistes» ont changé, les critères phylogénétiques ont succédé aux critères morphologiques; mais la visée est restée la même: réduire, en nommant, la profusion qui nous déborde. Cette nomination est conventionnelle; elle est normalisée au plan international. Mais la taxinomie ne s'intéresse qu'à des états et limite ses classifications à la seule hiérarchisation, ce qui n'est pas le cas de la terminologie, même si elle y accorde une place de choix.

Ensuite, Gabriel Otman confronte cette pratique scientifique aux classifications sémantiques par relations d'hyperonymie et d'hyponymie (pp. 43-54). Elles posent de nombreux problèmes que l'auteur examine, notamment à la lumière de la sémantique du prototype, qu'il utilise avec discernement, confrontant l'opérativité de cette théorie séduisante aux données objectives de son corpus. Confronté à la limite supérieure des hyperonymies, l'auteur, de façon pragmatique, isole pour le domaine de l'intelligence artificielle un ensemble de 17 hyperonymes ayant «valeur de primitives sémantiques» (p. 50).

Ces primitives n'ont d'utilité que replacées au sein d'un réseau, c'est-à-dire d'un ensemble relationnel. C'est dire qu'elles ne prennent sens qu'au sein de ces relations dans les réseaux sémantiques, auxquelles est consacré le troisième chapitre. Les relations «sorte de» et «partie-tout» sont examinées de leur hiérarchie et de leur héritage. Elles sont de nature conceptuelle et linguistique et doivent pouvoir être valablement reformulées dans la langue d'usage. On suit volontiers l'auteur lorsqu'il cherche à éviter la génération de phrases telles que «*tes nageoires rendent les poissons sauvages» (p. 57). De même, la réciprocité des relations est regardée de près, afin d'éviter des énoncés du type «*Bagheera est une espèce en danger» (p. 77).

L'étude très fine et complète de ces relations apporte des éclairages intéressants auxquels la perspective de modélisation doit beaucoup. L'auteur apporte notamment une contribution majeure à l'étude des relations partie-tout. Le choix d'un système à héritage de propriétés l'oblige à regarder de près le fonctionnement des diverses relations; par exemple: si $\mathrm{A}$ est une partie de $\mathrm{B}$ et $\mathrm{B}$ une partie de $\mathrm{C}$, peut-on en déduire que $\mathrm{A}$ est une partie de C? Pour obtenir des réseaux pertinents, l'auteur intègre l'héritage de prototype, qu'il distingue de l'hćritage de classes (p. 66). L'héritabilité cesse d'être la règle pour devenir une propriété pouvant s'exprimer de façon positive ou négative, ce qui permet alors de considérer la relation d'héritage comme transitive. 
Cela permet de rendre plus opérationnelle la relation «sorte de», au sein de laquelle sont distinguées l'appartenance (Socrate appartient à la classe des mortels) et l'inclusion (la classe des humains est incluse dans la classe des mortels). Seule la dernière, qui porte sur des classes, est transitive sans problème. Le mélange des genres conduit à des énoncés faux du type «*Socrate est nombreux» (p. 77). En revanche, dans un réseau où les propriétés sont héritées, cette relation ne peut être tenue pour réciproque : les vélos sont des moyens de transport, mais les moyens de transport ne possèdent pas de guidons.

La relation «partie de» unit le méronyme à au moins un holonyme, en raison des polyhiérarchies. Transitive au plan logique, elle l'est difficilement au plan linguistique: «? Le doigt est une partie du bras» (p. 82). Pour cerner la diversité des relations, l'auteur distingue cinq sous-classes : objet/élément, ensemble/membre, masse/portion, objet/constituant, activité / phase, chacune d'entre elles étant transitive. Les tests effectués montrent que les relations méronymiques mixtes ne sont transitives que dans un seul cas, lorsque se trouvent conjuguées les relations masse/portion et objet/constituant (pp. 88-92).

Ces mises au point sémantiques lui permettent de présenter son modèle de réseau sémantico-terminologique (RST) dans le chapitre cinq. La nature des relations prises en compte étant éclaircie, l'auteur s'intéresse à la représentation des graphes, qui connectent à la fois les termes modélisés, les traits distinctifs qui permettent de les opposer et leurs relations. Fidèle à son orientation, l'auteur considère qu'un terme «est reconnu et accepté par l'ensemble de la communauté linguistique de ce domaine de spécialité et, dans l'idéal, possède une définition consensuelle» ( $p .100$ ). Il retient dans son modèle six sortes de relations décrites. Elles sont de deux types: associatives (partie de, sorte de, fonction de, proximité de) et distinctives (contraste avec, équivalent de). Ces relations sont étudiées en détail et exemplifiées avec soin. La dernière (équivalent de) permet à l'auteur de prendre en compte tous les faits de synonymie dont il détaille les causes (p. 112). Cela lui permet de distinguer les équivalents qu'il retient par des traits expliquant cette synonymie (noms de marque, diachronie, diatopie, oral/écrit, métaphore, norme, etc.). Il s'ensuit une présentation de l'application résultant du modèle de RST, en l'occurrence une base de connaissances terminologiques (BCT).

L'auteur a appliqué son modèle à un Vocabulaire de l'intelligence artificielle, qu'il a fait paraître en 1991. Cela fait l'objet du chapitre cinq. Un champ aussi transdisciplinaire constitue un bon test pour un modèle. Il pose tout d'abord des problèmes de clôture : il est difficile de lui appliquer la notion de domaine, tant des terminologies endogène et exogène s'y interpénètrent (pp. 125-132). Fidèle à son optique post-classique, Gabriel Otman considère les terminologies endogènes comme étant «celles que l'on trouve dans les dictionnaires de spécialité» (p. 132). On voit mal comment, sur de telles bases, l'auteur ne pourrait pas marcher sur les traces de ses prédécesseurs, qu'il connaît bien et dont il parle en expert. Du reste, la critique est de peu de portée, car l'auteur fait montre d'une belle lucidité et son souci applicatif ne verse jamais dans le praticisme. Le terminologue reste linguiste, même dans la peau du terminographe.

Sa pratique terminographique utilise une fiche inspirée de celle conçue par Pierre Lerat pour le Centre de terminologie et de néologie, dont l'auteur fut un membre actif (pp. 139-142). Elle se distingue notamment par les champs «prédicat» (ou action typique) et «argument» (ou objet typique). La terminographie implique une phase de validation. Celle-ci revient à l'expert, personnage que l'on sait central dans la geste mythologique du peuple des terminologues. Gabriel Otman est conscient tout à la fois de son importance et des problèmes sociolinguistiques que soulève la conception que l'on se fait de son rôle. Renvoyant cette problématique à la socioterminologie, il opte pour la consultation à visée consensuelle d'un groupe d'experts (p. 144). 
L'auteur présente ensuite un traitement prototypique de son modèle, à partir du terme règle. Il teste ainsi la cohérence des graphes et dresse un bilan contrasté, livrant en détail les aspects positifs et négatifs. En fait, l'auteur ne relève que deux incohérences, mineures, dans les déductions établies par héritage des propriétés (p. 155). Cela prouve la sûreté de la conception, mais aussi la puissance d'un RST qui permet de repérer de telles erreurs. Ces deux caractères positifs sont obtenus au prix d'une économie du système de règles qui laisse dans l'ombre des traits définitoires importants. En effet, dans un RST, les termes ne sont définis que par leurs relations. Mais ces réserves mettent en évidence, plus que des faiblesses du système, les scrupules louables du concepteur.

Les conclusions, très positives, permettent à Gabriel Otman de consacrer son chapitre six à la présentation d'autres réalisations et projets. Il faut dire que l'auteur, présent dans le projet ICE et dans le PRC-IA, a déjà montré qu'il comptait, en France, parmi les chevilles ouvrières de la mise en synergie de la terminologie et des sciences cognitives. Il décrit ainsi Cogniterm, projet poursuivi à l'Université d'Ottawa par I. Meyer et D. Stucke, et le projet toulousain Matra Marconi Space. Il évoque également le traitement terminologique du TLF (projet ICE) et, concernant la recherche fondamentale, le groupe PRC-IA. Conscient des limites, sémantiques, formelles et informatiques, de l'outil qu'il a présenté, l'auteur laisse entrevoir, en conclusion, les applications possibles en matière d'autoapprentissage et de veille terminologique.

La rigueur dont fait preuve l'auteur dans la mise en place de son modèle, la lucidité dont il témoigne par rapport aux limites théoriques et pratiques du cadre qu'il s'est données sont à louer. Certes, des points peuvent être discutés: l'auteur reste un wüstérien éclairé. Peut-on penser et réaliser des applications informatiques tout en prenant en compte la dimension sociolinguistique de la communication spécialisée? L'auteur montre que cela serait souhaitable; il laisse prudemment à d'autres le soin d'en faire la démonstration. La question reste donc ouverte...

François GaUdin Université de Rouen, Rouen, France 